\title{
Near-haploid B lymphoblastic leukemia with an apparent hyperdiploid karyotype: the critical role of SNP analysis in establishing proper diagnosis
}

\author{
Sarah M. Choi • Peter Papenhausen • Gerald Wertheim • \\ Rebecca L. King
}

Received: 12 April 2013 / Accepted: 30 May 2013 / Published online: 8 June 2013

(C) Springer-Verlag Berlin Heidelberg 2013

\begin{abstract}
Molecular genetic analysis is indispensible in both classification and prognostication of hematopoietic malignancies. The evolution of genetic analysis from conventional cytogenetics to novel genomic technologies has increased our ability to detect subtle and nonconventional mutations in leukemia and lymphoma. Near haploidy, a subtype of hypodiploidy, is a rare entity that carries a particularly poor prognosis in B lymphoblastic leukemia (B-ALL). Nearhaploid B-ALL usually quickly evolves by doubling of the chromosome number, creating confusion with the prognostically favorable hyperdiploid B-ALL subtype. Here, we describe a case of B-ALL in which singlenucleotide polymorphism (SNP) microarray analysis was used to identify a hyperdiploid clone that had evolved from a presumptive, near-haploid clone. By conventional methods, this clone may have been easily misinterpreted as a common hyperdiploid clone. Given the extreme prognostic differences of the two clones, this distinction is especially critical to accurately guide therapy. In this case report and brief review, we discuss the role of SNP microarray analysis in the diagnosis and proper subclassification of B-ALL as well as its potential in the identification of genetic therapeutic targets.
\end{abstract}

S. M. Choi $\cdot$ G. Wertheim • R. L. King

Department of Pathology and Laboratory Medicine,

Perelman School of Medicine, University of Pennsylvania,

Philadelphia, PA, USA

S. M. Choi $\cdot$ G. Wertheim $•$ R. L. King $(\bowtie)$

Department of Pathology and Laboratory Medicine,

Children's Hospital of Philadelphia, 5 Main, 5140,

34th and Civic Center Blvd., Philadelphia, PA 19104, USA

e-mail: rebecca.king98@gmail.com

P. Papenhausen

Laboratory Corporation of Cytogenetics,

Research Triangle Park, NC, USA
Keywords SNP array · Hypodiploid · B lymphoblastic leukemia $\cdot$ Haploid $\cdot$ ALL

\section{Introduction}

B lymphoblastic leukemia (B-ALL) is the most common neoplasm in children, and despite modern advances in therapy that have vastly improved overall prognosis, approximately $20 \%$ of patients experience disease recurrence [1]. Numerous recurrent cytogenetic abnormalities have been described in B-ALL, many of which have been established as independent risk factors for survival in both children and adults [2-13]. Included among these is chromosome number, which provides important prognostic information that frequently influences management of pediatric B-ALL. Those cases with high hyperdiploidy (51-65 chromosomes), especially trisomy of chromosomes 4,10 , and 17 , have a favorable prognosis in children [13]. In contrast, hypodiploidy ( $<46$ chromosomes), which is seen in $5-8 \%$ of B-ALL patients, affords an extremely unfavorable prognosis [2, 4-6, 13, 14]. Hypodiploid B-ALL can be further subdivided based on degree of aneuploidy into near-haploid (23-29 chromosomes), low-hypodiploid (33-39 chromosomes), and high-hypodiploid (40-45 chromosomes) groups [14-16].

B-ALL with near-haploid or low-hypodiploid karyotypes have a propensity to undergo duplication of their entire chromosome complement, resulting in a chromosome number of 50-78 and thus complicating the distinction from a hyperdiploid karyotype [17]. Given the prognostic and therapeutic differences between hyperdiploid and hypodiploid B-ALL, awareness of this phenomenon, and the ability to distinguish these two scenarios, is essential.

While conventional chromosome analysis and fluorescence in situ hybridization (FISH) have been the traditional 
standards for defining chromosome number and identifying recurrent translocations in leukemic cells, the introduction of newer molecular technologies such as whole genome singlenucleotide polymorphism (SNP) microarray analysis has led to a greater understanding of the genetics of these neoplasms [7, 13, 17, 18]. High-density SNP analysis, which simultaneously measures allele-specific copy number at approximately one million unique loci, is particularly useful for analyzing neoplastic lesions in an unbiased, comprehensive manner. Importantly, SNP arrays can detect both copy number changes as well as copy number neutral loss of heterozygosity, which cannot be detected using traditional karyotyping [7, 12, 18-20]. Recent studies using this technology in cases of ALL have identified several new cryptic abnormalities that have not been detected using standard methodology [7, 12, 19, 21]. By correlating these abnormalities with clinically relevant information, these studies demonstrate that SNP analysis can be used in identifying clinically significant patient subgroups as well as in identifying possible therapeutic targets $[7,19,21,22]$.

Here, we describe a case of pediatric B lymphoblastic leukemia with a hyperdiploid karyotype that has evolved from a near-haploid clone that was not detectable by conventional cytogenetics. This case illustrates the limitations and possible pitfalls of conventional cytogenetic methods and highlights one role for SNP array analysis in establishing accurate genetic subclassification of this neoplasm.

\section{Materials and methods}

Pathologic evaluation and bone marrow studies

Bone marrow aspirates were prepared and stained with a Wright-Giemsa stain. Flow cytometric analysis was performed on bone marrow samples by CPA Laboratories (Louisville, KY) per their protocol using antibodies to $\mathrm{CD}$ 2, CD3, CD4, CD5, CD7, CD8, CD10, CD11c, CD13, CD14, CD19, CD20, CD23, CD25, CD33, CD34, CD38, CD45, CD56, CD117, CD138, HLA-DR, kappa surface light chain, and lambda surface light chain.

\section{Cytogenetic and FISH analysis}

Karyotyping was performed by Integrated Oncology, Laboratory Corporation of America Holdings (Brentwood, $\mathrm{TN}$ ) on bone marrow aspirates using standard protocols. Interphase FISH analysis on bone marrow aspirates was performed by Integrated Oncology, Laboratory Corporation of America Holdings (Brentwood, TN) per their protocols using the following probes: CEN 4, 10, and 17; ETV6(TEL)/RUNX1(AML1); MLL; BCR/ABL; p16(CDKN2A); and TCF3(E2A).
SNP microarray analysis

SNP microarray analysis was performed by Integrated Oncology, Laboratory Corporation of America Holdings (Brentwood, TN). Briefly, SNP microarray analysis was performed using the Affymetrix CytoScan HD platform. A total of $250 \mathrm{ng}$ of genomic DNA was extracted, digested with NspI, ligated to NSPI adaptors, and amplified using Titanium Taq with a GeneAmp PCR System 9700. PCR products were purified using AMPure beads and quantified using NAnoDrop 8000. Purified DNA was fragmented, biotin-labeled, and hybridized to the Affymetrix CytoScan HD GeneChip. Data were analyzed using Chromosome Analysis Suite and were based on the GRCh37/hg19 assembly.

\section{Clinical history and results}

Clinical history, bone marrow, and flow cytometry results

The patient is a 10 -year-old, previously healthy boy who presented to his physician complaining of fatigue and bone pain in September 2012. There was no reported family history of childhood cancer. A complete blood count was performed and demonstrated a WBC count of 17,500 cells $/ \mu \mathrm{L}, 60 \%$ of which were blasts, with concomitant anemia and thrombocytopenia. A bone marrow study was then performed, demonstrating a hypercellular marrow with greater than $90 \%$ blasts (Fig. 1). Flow cytometry revealed the blasts to be of $\mathrm{B}$ cell origin with the following immunophenotype: positive for CD19, CD10, CD22, CD34, CD38, HLA-DR, and TdT; negative for CD3, CD20, sIgM, cIgM, MPO, kappa, lambda, and other myeloid and $\mathrm{T}$ cell antigens. These combined findings were diagnostic of B lymphoblastic leukemia. There was no evidence of central nervous system or testicular involvement.

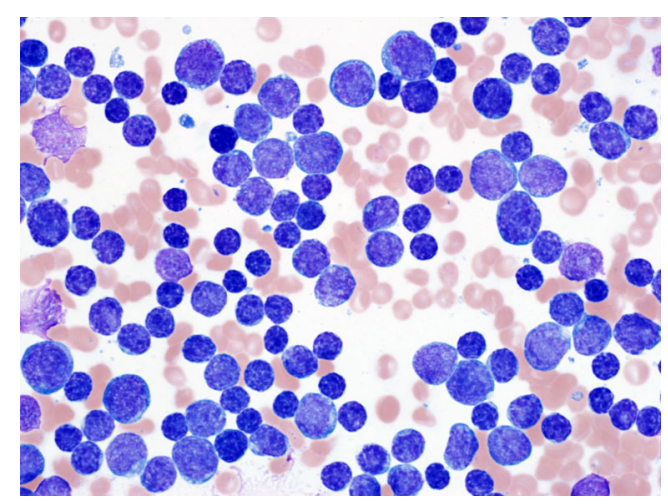

Fig. 1 Bone marrow aspirate demonstrates numerous small- to mediumsized lymphoblasts. Wright-Giemsa stain, $\times 1,000$ 


\section{Cytogenetic and FISH analysis}

Conventional metaphase analysis revealed the following karyotype: $52, \mathrm{XY},+\mathrm{X},+\mathrm{Y},+14,+14,+21,+21[\mathrm{cp} 20]$. The gain of the $\mathrm{Y}$ chromosome as well as tetrasomy for chromosomes 14 and 21 was suggestive of duplicated near-haploid state, although no such metaphases were identified (Fig. 2).

FISH studies demonstrated that $95 \%$ of nuclei possessed four copies of the RUNX1 locus on chromosome $21 \mathrm{q} 22$ (Fig. 3). No ETV6/RUNX1, MLL, BCR/ABL, or TCF3 gene rearrangements were detected. No deletion of $p 16$ was seen. Centromeric probes for chromosomes 4, 10, and 17 revealed two copies of each in $100 \%$ of cells. Consistent with the metaphase karyotype, single-copy signals consistent with near haploidy were not detected.

\section{SNP microarray analysis demonstrates near haploidy}

Consistent with karyotype analysis, whole-genome SNP microarray analysis demonstrated an abnormal clone with a chromosomal complement of a whole number multiple of 26 chromosomes (i.e., 52 chromosomes). Given the allele dosage patterns showing either diploidy or tetraploidy, this is consistent with an original clone that had 26 chromosomes including two copies of chromosomes 14 and 21 and a single copy of all other chromosomes, including the $\mathrm{X}$ and $\mathrm{Y}$. The presence of heterozygosity in chromosomes 14 and 21 with a copy number dosage of 4 (Fig. 4a), combined with the loss of heterozygosity in the remaining diploid chromosomes (Fig. 4b), is a conclusive evidence of near-haploid origin.

\section{Discussion}

This is an interesting case of childhood B-ALL that required SNP array data for proper subclassification and designation of the patient to the appropriate prognostic subgroup. On initial review, the karyotype appears hyperdiploid with 52

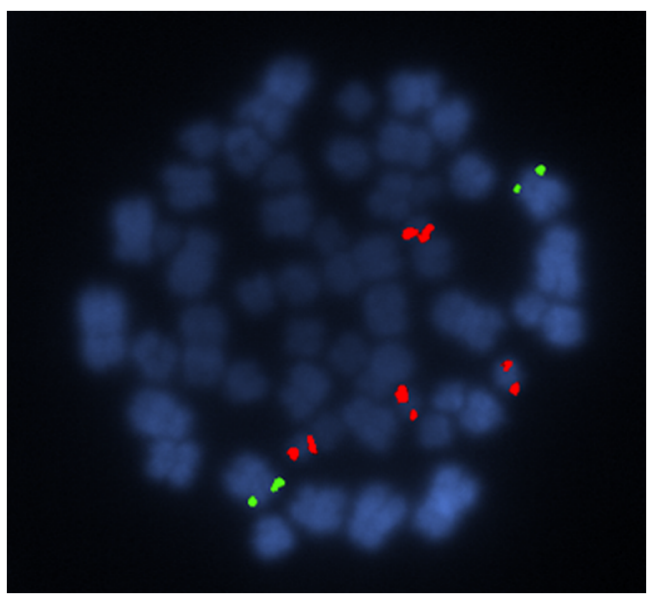

Fig. 3 ETV6/RUNX1 (TEL/AML1) FISH. Four RUNX1 signals (chromosome 21, red) and two ETV6 signals (chromosome 12, green) are identified

chromosomes and suggests that the patient should be riskstratified into the relatively common and prognostically favorable hyperdiploid subgroup. There are several features of the patient's karyotype, however, that are not entirely consistent with the usual hyperdiploid profile. In common hyperdiploidy, gain of chromosome $\mathrm{X}$ but not chromosome $\mathrm{Y}$ is seen, whereas this patient showed diploid for both. Furthermore, unlike this patient who had tetrasomy for chromosomes 14 and 21, standard hyperdiploid clones usually have tetrasomy 21 without other tetrasomic chromosomes [21]. Typical hyperdiploid clones also show gain of chromosomes 4,10 , and 17 , and the absence of this finding, as seen in this case, would be highly unusual $[10,13]$. Thus, the karyotypic findings in this case suggest evolution of a nearhaploid clone in spite of the fact that such a clone was not detected by conventional cytogenetics.

Hypodiploidy, and especially near haploidy, confer an extremely poor prognosis and high risk of relapse in BALL $[8,15]$. In children, the 3-year event-free survival (EFS) rate for the low-hypodiploid and near-haploid groups
Fig. 2 Representative karyotype showing tetraploidy of chromosomes 14 and 21 and diploidy of all remaining chromosomes including $\mathrm{X}$ and $\mathrm{Y}$

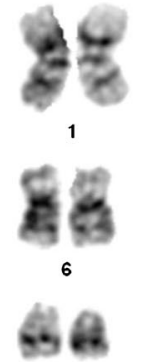

13

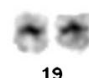

19

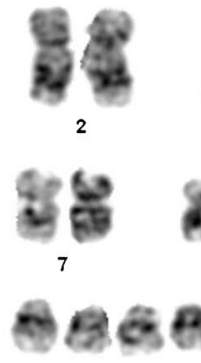

14

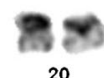

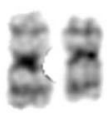

3

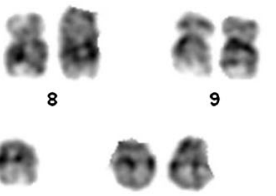

15

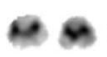

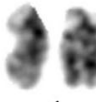

4

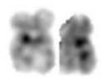

10
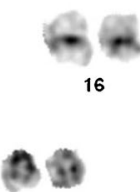

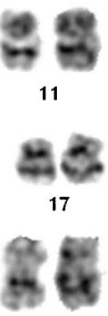

$\mathrm{x}$

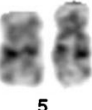
5

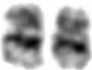

12

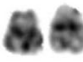

18

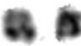

Y

$52, X Y,+X,+Y,+14,+14,+21,+21[c p 20]$ 
Fig. 4 SNP analysis of representative disomic and tetrasomic chromosomes. The top y-axis histogram represents copy number state and the bottom y-axis histogram segregates allele calls based on dosage $(A=+0.5, B=-0.5)$. a SNP analysis of chromosome 14. The top histogram shows signal levels consistent with four copies of all loci analyzed. The bottom histogram demonstrates the midline (0 line) heterozygosity line throughout the analyzed region. b SNP analysis of chromosome 2. The top histogram shows signal levels consistent with two copies of all loci analyzed. The bottom histogram demonstrates homozygosity at the vast majority of loci analyzed, illustrated by the absence of $\mathrm{AB}$ alleles on the center (0) line. This is consistent with loss of heterozygosity

\section{a}

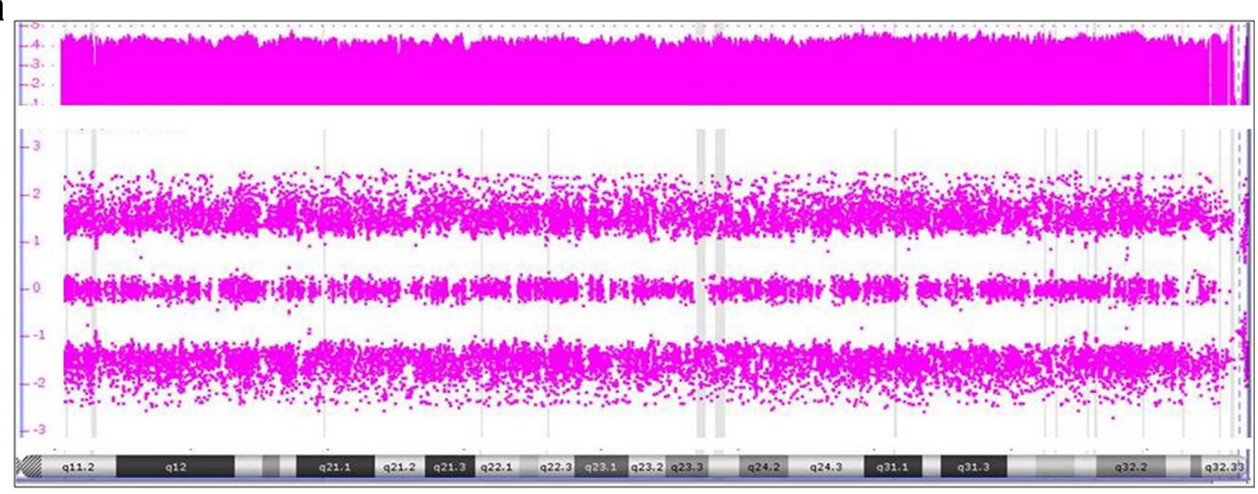

b

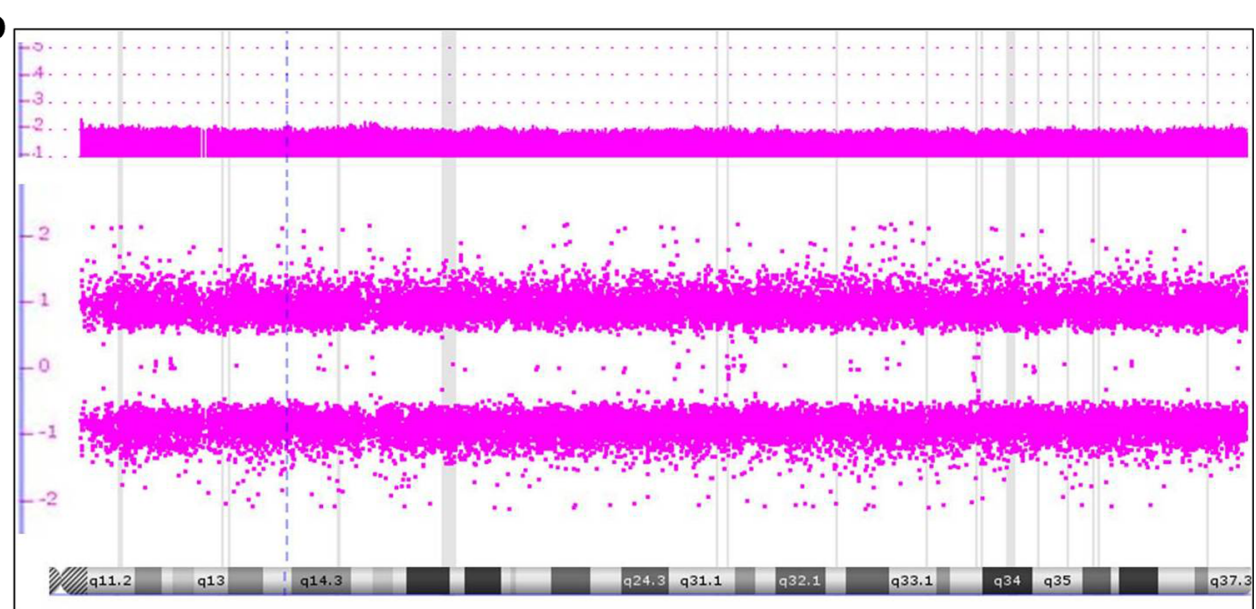

is $30 \%$, compared with $66 \%$ in the high-hypodiploid group $[9,10,15,16,23,24]$. In contrast, hyperdiploid B-ALL patients have up to an $80 \% 5$-year EFS [25]. Thus, the distinction between a hyperdiploid clone and a nearhaploid clone with evolution is critical both for the prediction of prognosis and more importantly for the guidance of therapy in these children. Current recommendations are that children with near-haploid B-ALL proceed directly to allogeneic stem cell transplant at the first complete remission [26], whereas treatment for hyperdiploid B-ALL is a more standard multiphase chemotherapy regimen.

Although hypodiploidy can be seen in 5-8 \% of B-ALL, cases that fall into the near-haploid group are extremely rare, accounting for $<1 \%$ of B-ALL $[12,13,15,27]$. This frequency has likely been underestimated due to the propensity of these clones to undergo clonal evolution by chromosomal duplication resulting in an apparently hyperdiploid karyotype $[15,17,28]$. This genetic phenomenon highlights an important limitation of karyotyping and FISH in cases where the original clone is no longer present, as is often the case in near haploidy and severe hypodiploidy. Thus, as in this case, apparent hyperdiploidy detected via conventional cytogenetics can potentially mask an underlying hypodiploid or nearhaploid state $[15,17,28]$. As demonstrated here, the use of SNP array confirmed the suspicion of a doubled, near-haploid clone that was not directly detected in conventional karyotypic or FISH analyses.

Near-haploid B-ALL is characterized by several unique genetic attributes including the rarity of concurrent structural abnormalities, preferential retention of chromosomes 14, 18, 21 , and $\mathrm{X} / \mathrm{Y}$, as well as frequent duplication of the nearhaploid genome [15-17]. In a recent case series of nearhaploid B-ALL, chromosome 21 was retained in $8 / 8$ cases, followed by chromosome 14 in $6 / 8$ and $\mathrm{X} / \mathrm{Y}$ in $5 / 8$, thus highlighting the nonrandom nature of chromosome retention [29]. Our case highlights a very typical example of the uncommon near-haploid B-ALL, made especially diagnostically challenging by the lack of the original hypodiploid clone.

Low-hypodiploid B-ALL has a tendency to exhibit retention of the same chromosomes as in near-haploid B-ALL but, in addition, often shows retention of chromosomes 1, 11, and 19. Additionally, duplication of chromosome number is also frequently observed in low hypodiploidy, yet unlike near haploidy, this entity frequently exhibits structural abnormalities, including translocations. High-hypodiploid B-ALL is quite distinct from the former two groups in that reductions in chromosomal number are typically due to loss of whole chromosome or unbalanced translocations. Most of these patients have complex karyotypes and do not show duplicated hyperdiploid states [15]. 
Due to the relative rarity of hypodiploid B-ALL, little is known about the specific genetic features that confer a predilection for disease relapse. Whole-genome and FISH analysis of relapsed B-ALL patients suggest that the relapsed clones represent subpopulations of the original leukemia that have undergone selection via the patient's therapeutic regimen [22, 30]. In the cases of ALL at diagnosis when near haploidy was initially observed within a mixture of other karyotypes, the near-haploid clone later predominated in the relapsed marrow [22]. Retention of particular chromosomes in near haploidy might affect the expression of genes that ultimately confer a survival or proliferative advantage to these cells or induce drug resistance [31-33]. Haploinsufficiency of certain genes on monosomic chromosomes has also been suggested to potentially contribute to the transformation and relapse in both near-haploid and low-hypodiploid clones [28]. Additional investigation into the identity of the affected genes may help elucidate the mechanism by which near haploidy and hypodiploidy lead to treatment failure and relapse.

Recent sequencing studies reveal that many patients with near-haploid B-ALL have deletions and mutations affecting the Ras signaling pathway as well as specific members of the IKAROS lymphoid transcription factor family $[8,11,12,34]$. Of note, this case did not show a deletion in the IKAROS gene. In addition, these studies have demonstrated that nearhaploid cases show mutations in a different subset of genes than those seen in other low-hypodiploid cases $[8,11,12$, 34]. These genetic studies clearly demonstrate the potential for identification of targeted therapies and the further subclassification of this poorly understood subtype of B-ALL.

In summary, this case represents a typical example of the genetic features seen in near-haploid B-ALL and the potential for misidentifying these cases, as many present as doubled, near-haploid clones. As such, SNP array analysis, which can easily distinguish heterozygosity from homozygosity on a genomic scale, provides critical information to aid in this distinction.

Conflict of interest The authors declare that they have no conflict of interest.

\section{References}

1. Pui CH, Relling MV, Downing JR (2004) Acute lymphoblastic leukemia. N Engl J Med 350(15):1535-1548

2. Chessels JM, Swansbury GJ, Reeves B et al (1997) Cytogenetics and prognosis in childhood lymphoblastic leukemia: results of MRC UKALL X. Br J Haematol 99:93-100

3. Secker-Walker LM, Lawler SD, Hardisty RM (1978) Prognostic implications of chromosomal findings in acute lymphoblastic leukaemia at diagnosis. BMJ 2:1529-1530

4. Pui CH, Williams DL, Raimondi SC et al (1987) Hypodiploidy is associated with a poor prognosis in childhood acute lymphoblastic leukemia. Blood 70:247-253
5. Trueworthy R, Shuster J, Look T et al (1992) Ploidy of lymphoblasts is the strongest predictor of treatment outcome in Bprogenitor cell acute lymphoblastic leukemia of childhood: a Pediatric Oncology Group study. J Clin Oncol 10:606-613

6. Heerema NA, Nachman JB, Sather HN et al (1999) Hypodiploidy with less than 45 chromosomes confers adverse risk in childhood acute lymphoblastic leukemia: a report from the children's cancer group. Blood 94:4036-4045

7. Okamoto R, Ogawa S, Nowak D et al (2010) Genomic profiling of adult acute lymphoblastic leukemia by single nucleotide polymorphism oligonucleotide microarray and comparison to pediatric acute lymphoblastic leukemia. Haematologica 95(9):1481-1488

8. Loh ML, Mullighan CG (2012) Advances in the genetics of highrisk childhood B-progenitor acute lymphoblastic leukemia and juvenile myelomonocytic leukemia: implications for therapy. Clin Cancer Res 18(10):2754-2767

9. Forestier E, Johansson B, Gustafsson G et al (2000) Prognostic impact of karyotypic findings in childhood acute lymphoblastic leukaemia: a Nordic series comparing two treatment periods. For the Nordic Society of Paediatric Haematology and Oncology (NOPHO) Leukaemia Cytogenetic Study Group. Br J Haematol 110:147-153

10. Moorman AV, Harrison CJ, Buck GA et al (2007) Karyotype is an independent prognostic factor in adult acute lymphoblastic leukemia (ALL): analysis of cytogenetic data from patients treated on the Medical Research Council (MRC) UKALLXII/Eastern Cooperative Oncology Group (ECOG) 2993 trial. Blood 109:3189-3197

11. Mullighan CG (2012) The molecular genetic makeup of acute lymphoblastic leukemia. Hematol Am Soc Hematol Educ Program 2012:389-396

12. Holmfeldt L, Wei L, Diaz-Flores E et al (2013) The genomic landscape of hypodiploid acute lymphoblastic leukemia. Nat Genet 45(3):242-252

13. Swerdlow S, Campo E, Harris N, Jaffe E, Pileri S, Stein H, Thiele J, Vardiman J (eds) (2008) WHO classification of tumours of haematopoietic and lymphoid tissues. IARC, Lyon

14. Nachman JB, Heerema NA, Sather H et al (2007) Outcome of treatment in children with hypodiploid acute lymphoblastic leukemia. Blood 110:1112-1115

15. Harrison CJ, Moorman AV, Broadfield ZJ et al (2004) Three distinct subgroups of hypodiploidy in acute lymphoblastic leukaemia. Br J Haematol 125:552-559

16. Pui CH, Carroll AJ, Raimondi SC et al (1990) Clinical presentation, karyotypic characterization, and treatment outcome of childhood acute lymphoblastic leukemia with a near-haploid or hypodiploid less than 45 line. Blood 75:1170-1177

17. Onodera N, McCabe NR, Nachman JB et al (1992) Hyperdiploidy arising from near-haploidy in childhood acute lymphoblastic leukemia. Gene Chromosome Cancer 4:331-336

18. Van Loo P, Nilsen G, Nordgard SH et al (2012) Analyzing cancer samples with SNP arrays. Methods Mol Biol 802:57-72

19. Kawamata N, Ogawa S, Zimmermann M et al (2008) Molecular allelokaryotyping of pediatric acute lymphoblastic leukemias by high-resolution single nucleotide polymorphism oligonucleotide genomic microarray. Blood 111(2):776-784

20. Nannya Y, Sanada M, Nakazaki K et al (2005) A robust algorithm for copy number detection using high-density oligonucleotide single nucleotide polymorphism genotyping arrays. Cancer Res 65(14):6071-6079

21. Sunil SK, Prakash PN, Hariharan S et al (2006) Adult acute lymphoblastic leukemia with near haploidy, hyperdiploidy and $\mathrm{Ph}$ positive lines: a rare entity with poor prognosis. Leuk Lymphoma 47(3):561-563

22. Mullighan CG, Phillips LA, Su X et al (2008) Genomic analysis of the clonal origins of relapsed acute lymphoblastic leukemia. Science 322:1377-1380 
23. Hann I, Vora A, Harrison G et al (2001) Determinants of outcome after intensified therapy of childhood lymphoblastic leukaemia: results from Medical Research Council United Kingdom acute lymphoblastic leukaemia XI protocol. Br J Haematol 113:103-114

24. Charrin C, Thomas X, Ffrench M et al (2004) A report from the LALA-94 and LALA-SA groups on hypodiploidy with 30 to 39 chromosomes and near-triploidy: 2 possible expressions of a sole entity conferring poor prognosis in adult acute lymphoblastic leukemia (ALL). Blood 104:2444-2451

25. Moorman AV, Richards SM, Martineau M et al (2003) Outcome heterogeneity in childhood high-hyperdiploid acute lymphoblastic leukemia. Blood 102(8):2756-2762

26. Wayne AS, Baird K, Egeler RM (2010) Hematopoietic stem cell transplantation for leukemia. Pediatr Clin N Am 57(1):1-25

27. Chan LC, Ha SY, Ching LM et al (1994) Cytogenetics and immunophenotypes of childhood acute lymphoblastic leukemia in Hong Kong. Cancer Genet Cytogenet 76:118-124

28. Stark B, Jeison M, Gobuzov R et al (2001) Near haploid childhood acute lymphoblastic leukemia masked by hyperdiploid line: detection by fluorescence in situ hybridization. Cancer Genet Cytogenet 128:108-113
29. Safavi S, Forestier E, Golovleva I et al (2013) Loss of chromosomes is the primary event in near-haploid and low-hypodiploid acute lymphoblastic leukemia. Leukemia 27(1):248-250

30. van Delft FW, Horsley S, Colman S et al (2011) Clonal origins of relapse in ETV6-RUNX1 acute lymphoblastic leukemia. Blood 117(23):6247-6254

31. Ma SK, Chan GC, Wan TS et al (1998) Near-haploid common acute lymphoblastic leukaemia of childhood with a second hyperdiploid line: a DNA ploidy and fluorescence in-situ hybridization study. $\mathrm{Br}$ J Haematol 103:750-755

32. Redner A, Hegewisch S, Haimi J et al (1990) A study of multidrug resistance and cell kinetics in a child with near haploid acute lymphoblastic leukemia. Leuk Res 14:771-777

33. Choi S, Henderson MJ, Kwan E et al (2007) Relapse in children with acute lymphoblastic leukemia involving selection of a preexisting drug-resistant subclone. Blood 110(2):632-639

34. Holmfeldt L, Zhang J, Ma J, et al (2010) Genome-wide analysis of genetic alterations in hypodiploid acute lymphoblastic leukemia identifies a high frequency of mutations targeting the IKAROS gene family and Ras signaling. Blood [ASH Annual Meeting Abstracts] 116:411 